\title{
Parity violation in electron scattering
}

\author{
S. Kowalski ${ }^{\mathrm{a}}$ \\ Laboratory for Nuclear Science and Department of Physics, Massachusetts Institute of Technology, Cambridge, MA, 02139, USA \\ / \\ Published online: 26 May 2006 - C Società Italiana di Fisica / Springer-Verlag 2006
}

\begin{abstract}
Parity-violating electron scattering has been a very useful tool for probing the structure of neutral currents and providing detailed information on electroweak form factors. A pioneering SLAC measurement in the mid-70s provided an important early test of the Standard Model. Modern electron accelerators provide high-intensity $(>100 \mu \mathrm{A})$, CW beams with polarizations as high as $85 \%$. Experiments such as SAMPLE, A4, HAPPEX and G0 have exploited these capabilities and obtained new information on electroweak strange form factors in the $Q^{2}$ range of $0.1-1.0(\mathrm{GeV} / c)^{2}$. That activity continues. Other experiments are designed to provide stringent tests of the Standard Model. E-158 at SLAC recently measured the weak charge of the electron. $Q_{\text {weak }}$ is a challenging new experiment at JLAB which is designed to measure the weak charge of the proton. This will probe for physics beyond the Standard Model corresponding to energy scales of more than $5 \mathrm{TeV}$.
\end{abstract}

PACS. 12.15.-y Electroweak interactions - 14.20.Dh Protons and neutrons - 21.10.Gv Mass and neutron distributions

\section{Introduction}

The electromagnetic probe is one of our most important tools for probing nucleon and nuclear structure and dynamics. Modern accelerators use electron scattering to probe distances of less than $1 \mathrm{fm}$ where signatures of quark degrees of freedom are expected to be observable. A new generation of electron accelerators, $0.5-6 \mathrm{GeV}$, with $\mathrm{CW}$ capability and intense polarized beams have become operational over the past decade. This has enhanced our capability for doing coincidence experiments and other measurements by more than two orders of magnitude. During this time, longitudinally polarized electrons have emerged as a very important new tool to study nucleon and nuclear structure. They have provided precise new information on nuclear electromagnetic form factors and other nuclear structure functions.

An important class of experiments using polarized electrons has been the study of parity violation. Parityviolating electron scattering involves the scattering of longitudinally polarized electrons from an unpolarized target. A change in counting rate resulting from a reversal of the beam helicity is a signal of a parity non-conserving effect. At high energies the SLAC parity violation experiment provided a crucial understanding of electro-weak processes and a precise measurement of the weak mixing angle, $\sin ^{2} \theta_{W}$. At low energies the first parity violation experiments were carried out at MIT-Bates and Mainz.

\footnotetext{
a e-mail: sbk@mit.edu
}

These were designed as tests of the Standard Model. During the past decade there has been an extensive program of such parity violating experiments at MIT-Bates, JLAB and Mainz. The goal of these parity-violating experiments currently is three-fold:

- study the strangeness content of the nucleon,

- measure the neutron density distribution in a heavy nucleus

and

- sensitive tests of the Standard Model.

In this paper, we will discuss and report on the recent progress of parity violation experiments in these three broad physics areas. Results and future prospects of the different experiments will be presented. The Mainz A4 experiment will not be discussed since it is the subject of another contribution to these proceedings.

\section{Physics program}

\subsection{Nuclear structure: strange quarks}

The proton is made up of three valence quarks, uud, and a sea of gluons and $q \bar{q}$ pairs all of which contribute to its electromagnetic properties at short distance scales. It was realized [1] that a measurement of the parity-violating asymmetry arising from the interference between the electromagnetic and neutral current amplitudes would allow us to extract the contributions of strange quarks to the 
ground state charge and magnetization distributions (e.g., magnetic moment) of the nucleon.

There have been many theoretical estimates of strange quark contributions to nucleon properties. These include both phenomenological models and lattice-gauge calculations. Separation of strange quark contributions to nucleon currents was developed by Kaplan and Manohar [2]. The parity-violating asymmetry [3] for scattering longitudinally polarized electrons from a proton can be written as

$$
\begin{aligned}
& A_{P V}=\frac{-G_{F}}{4 \pi \alpha \sqrt{2}} . \\
& \cdot\left[\frac{\varepsilon G_{E}^{p} G_{E}^{Z}+\tau G_{M}^{p} G_{M}^{Z}-\varepsilon^{\prime}\left(1-4 \sin ^{2} \theta_{W}\right) G_{M}^{p} G_{A}^{e}}{\varepsilon\left(G_{E}^{p}\right)^{2}+\tau\left(G_{M}^{p}\right)^{2}}\right] .
\end{aligned}
$$

The three terms in $A_{P V}$ arise as a result of the interference between the electromagnetic and weak interactions. The terms contain bilinear products of electromagnetic and weak form factors. $G_{E, M}^{p}$ are the form factors associated with the distribution of the protons charge and magnetism. The weak form factors $G_{E, M}^{Z}$ and $G_{A}^{e}$ contain contributions from strange quarks, $G_{E, M}^{s}$.

The kinematic factors depend upon specifics of an experiment. They are chosen to enhance the relative sensitivity of individual terms to $A_{P V}$. The first two terms are important at forward angles and the last two at backward angles:

$$
\begin{gathered}
\tau=Q^{2} / 4 M_{p}^{2}, \\
\varepsilon=\left[1+2(1+\tau) \tan ^{2}\left(\theta_{e} / 2\right)\right]^{-1}
\end{gathered}
$$

and

$$
\varepsilon^{\prime}=\sqrt{\left(1-\varepsilon^{2}\right) \tau(1+\tau)}
$$

\section{SAMPLE}

SAMPLE [4] at MIT-Bates was the first experiment to use parity violation as a probe for strange quarks in the proton. A longitudinally polarized electron beam of $200 \mathrm{MeV}$ was incident on a liquid hydrogen or deuterium target. The polarized electrons were produced using a bulk GaAs crystal resulting in an average polarization of $36 \%$. The linac produces a pulsed beam of $25 \mu$ s duration at a repetition rate of $600 \mathrm{~Hz}$. The beam current was $40 \mu \mathrm{A}$. The helicity of the beam is changed randomly pulse-by-pulse and in addition a half-wave plate can be inserted to change the overall sign of the helicity.

The scattered electrons were detected in a large solid angle $(1.5 \mathrm{sr})$ air Cerenkov detector spanning angles between $130^{\circ}$ and $170^{\circ}$. At backward angles SAMPLE is mostly sensitive to $G_{M}^{s}$ and $G_{A}^{e}$. Figure 1 shows one of the 10 detector modules which are placed A symmetrically about the beam axis. The Cherenkov light is focused by an ellipsoidal mirror unto a phototube. The integrated light is proportional to the scattered electron rate of about $10^{8} \mathrm{~s}^{-1}$ in a beam pulse.

A shutter located in front of the phototube could be closed providing a measurement of the background originating from neutrons and charged particles. Tight control of helicity-correlated effects on the properties of the beam

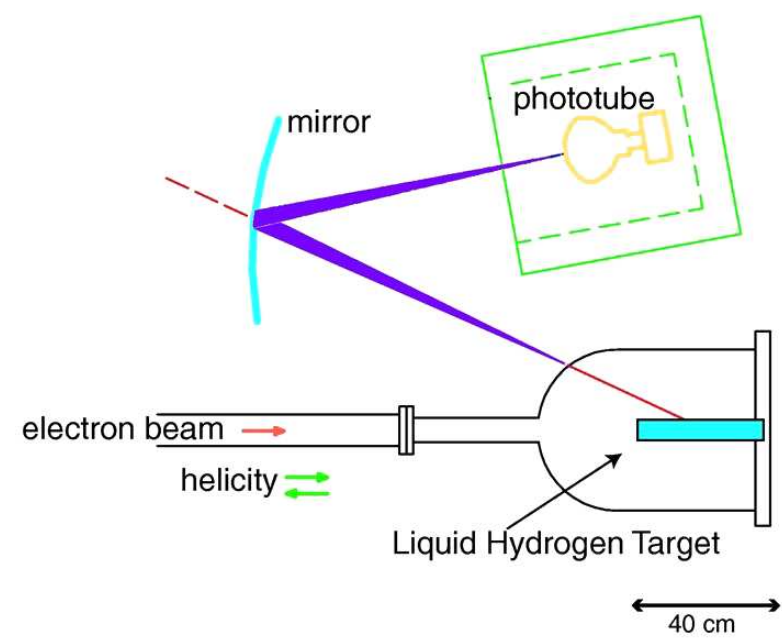

Fig. 1. A schematic view of one module of the SAMPLE experimental apparatus. Ten mirror-phototube pairs are placed asymmetrically about the beam axis.

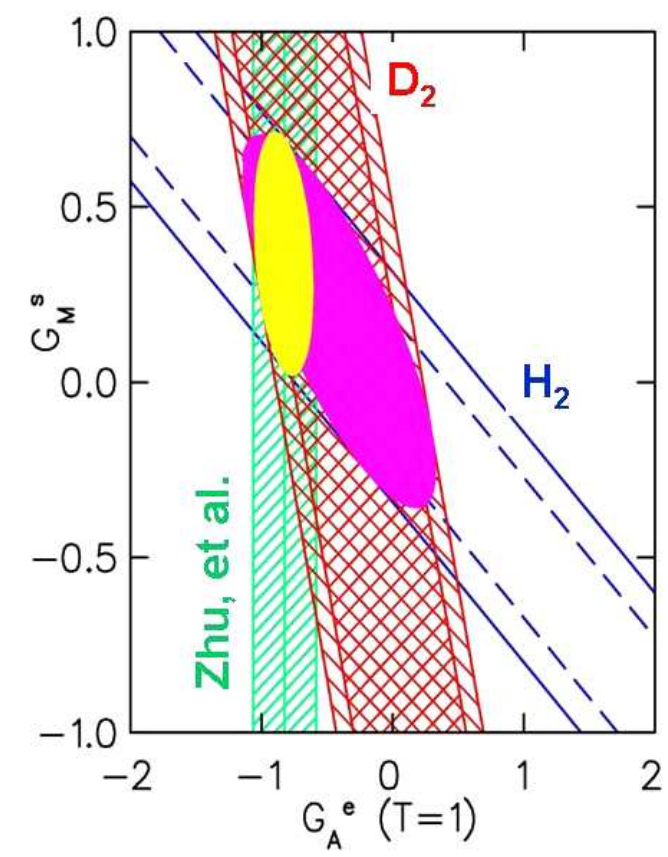

Fig. 2. Uncertainty bands of $G_{M}^{s}$ vs. $G_{A}^{e}$ at $Q^{2}=0.1(\mathrm{GeV} / c)^{2}$ for the SAMPLE experiment in both hydrogen and deuterium. Also shown is the uncertainty band of the theoretical expectation for $G_{A}^{e}$.

was critical to the success of the experiment. Several feedback systems were used to minimize such effects. These included energy, beam position, angle, and intensity. All parity experiments implement similar feedback controls.

Experiments were carried out at $200 \mathrm{MeV}$ on both hydrogen and deuterium targets. In addition a measurement at $125 \mathrm{MeV}$ was also made on a deuterium target. The two targets in principle allow a separation of $G_{M}^{s}$ and $G_{A}^{e}$. The results for both hydrogen and deuterium are summarized 
in fig. 2. Using a theoretical prediction for $G_{A}^{e}(T=1)$ one can extract a value for $G_{M}^{s}$ :

$$
G_{M}^{s}\left(Q^{2}=0.1(\mathrm{GeV} / c)^{2}\right)=0.37 \pm 20 \pm 0.26 \pm 0.07 .
$$

The experiment favors a small positive value for the magnetic moment contribution, $\mu_{s}$, which is at variance with most theoretical predictions.

\section{HAPPEX}

HAPPEX at JLAB was the second experiment designed to look for strange quarks using parity violating electron scattering. The first measurement was carried out at $Q^{2}=0.48(\mathrm{GeV} / c)^{2}$. A $3.2 \mathrm{GeV}$ beam of polarized electrons was incident on a liquid hydrogen target. The scattered electrons were detected in the pair of high resolution spectrometers in Hall-A at a scattering angle of $12.5^{\circ}$. Beam currents up to $100 \mu \mathrm{A}$ were used. The Cherenkov light from a lead-lucite sandwich was integrated over the duration of the helicity window. Beam helicity was reversed at $30 \mathrm{~Hz}$. Beam polarization was $38 \%$ in the first run and improved to $70 \%$ in the second run. It was measured with both Moeller and Compton polarimeters. Tight control of helicity correlated systematics was similar to those used in the SAMPLE experiment.

The experiment is sensitive to both $G_{E}^{s}$ and $G_{M}^{s}$. They extracted [5] a linear combination of strange form factors,

$$
G_{E}^{s}+0.392 G_{M}^{s}=0.014 \pm 0.020 \pm 0.010 \text {. }
$$

In 2004, a second HAPPEX measurement was carried out at $Q^{2}=0.10(\mathrm{GeV} / c)^{2}$. Measurements were made on both ${ }^{1} \mathrm{H}$ and ${ }^{4} \mathrm{He}$ targets. Helium is a special target since the nuclear spin $\mathrm{I}=0$. It has sensitivity only to $G_{E}^{s}$. Combining both the hydrogen and helium results allows a direct extraction of $G_{E}^{s}$ and $G_{M}^{s}$.

A $3.0 \mathrm{GeV}$ polarized electron beam was incident on the target. The scattered electrons were detected in the pair of high resolution spectrometers in Hall-A at a scattering angle of $6^{\circ}$. A pair of septum magnets in front of the spectrometers made this feasible. Beam currents of up to $35 \mathrm{~A}$ were used. Total absorption detectors were used to detect the scattered electrons.

The experiment on hydrogen yielded [6] the strange form factor combination,

$$
G_{E}^{s}+0.08 G_{M}^{s}=0.030 \pm 0.025 \pm 0.006 .
$$

The measurement on ${ }^{4} \mathrm{He}$ yielded [7],

$$
G_{E}^{s}=-0.038 \pm 0.042 \pm 0.010 .
$$

Both measurements are consistent with zero. The combined hydrogen and helium HAPPEX results were consistent with no evidence for strange quarks at $Q^{2}=$ $0.1(\mathrm{GeV} / c)^{2}$. The results are summarized in fig. 3 .

Following the above Phase-I measurements, a second experiment on both hydrogen and helium was completed in 2005. These results, not yet available, when analyzed are projected to have error bars which are $1 / 3$ those obtained so far. This will be a very significant improvement.

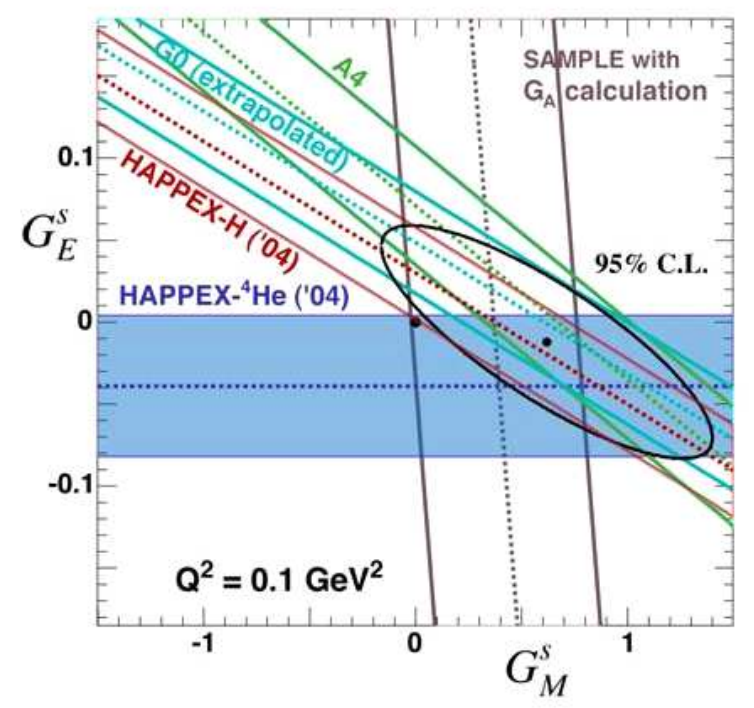

Fig. 3. The four $A_{P V}$ measurements at $Q^{2}=0.1(\mathrm{GeV} / \mathrm{c})^{2}$ are shown, with shaded bands representing 1-sigma combined statistical and systematic uncertainty. Also shown is the combined $95 \%$ C.L. ellipse from all four measurements.

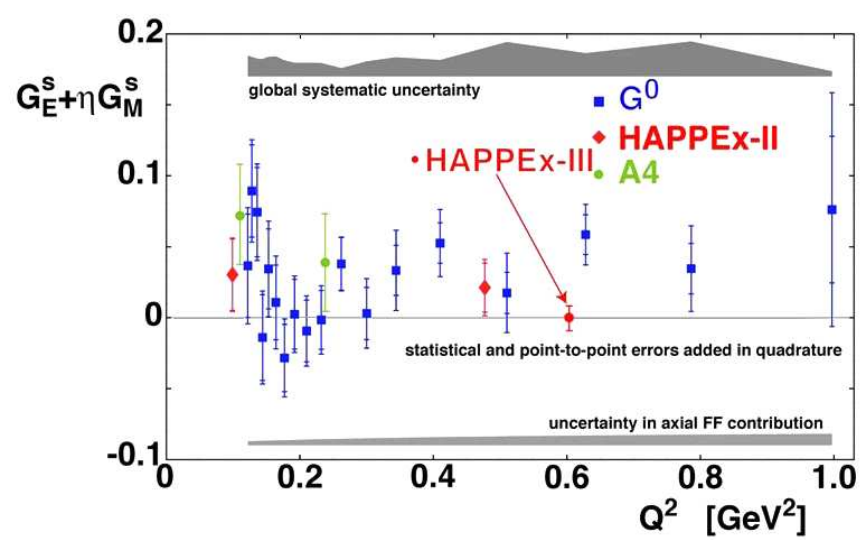

Fig. 4. The combination $G_{E}^{s}+\eta G_{M}^{s}$ for the HAPPEX, A4 and G0 experiments. Also shown at $Q^{2}=0.6(\mathrm{GeV} / c)^{2}$ the projected uncertainty for a future HAPPEX-III measurement.

In a future experiment HAPPEX is planning a measurement at $Q^{2}=0.6(\mathrm{GeV} / c)^{2}$. Figure 4 shows the expected uncertainty for this measurement. We expect to run this experiment in 2008-09.

3. G0

G0 is another ambitious JLAB parity violation experiment. Its goal is to search for evidence of strange quarks in the proton. It is designed to make both forward and backward angle measurements in the $Q^{2}$ range, $0.2-$ $1.0(\mathrm{GeV} / c)^{2}$.

G0 is an 8 sector superconducting toroidal spectrometer. A $3.0 \mathrm{GeV}$ electron beam was incident on a liquid hydrogen target. The beam current was $40 \mu \mathrm{A}$. The detector is designed to detect the recoil protons (fig. 5). It is segmented along the focal plane (16) so that each segment 


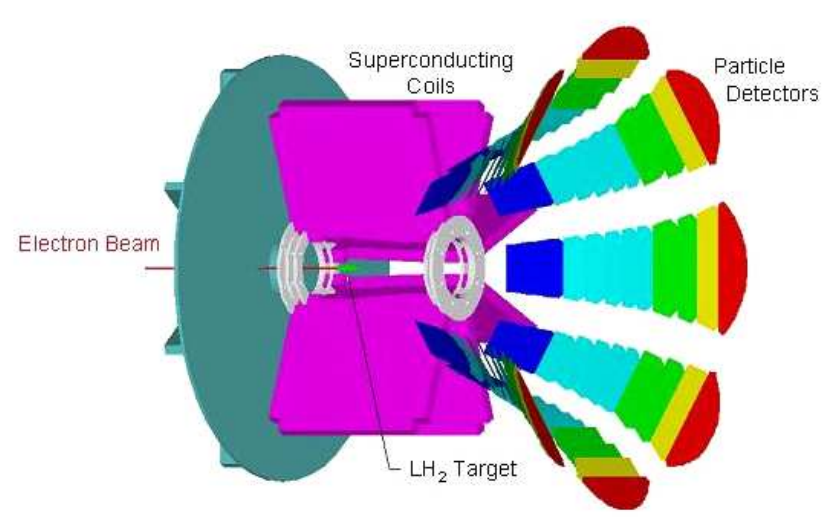

Fig. 5. Layout of the G0 spectrometer system. Shown are the superconducting toroidal magnet and the segmented scintillator detector array.

measures a different $Q^{2}$ range. The beam duty factor was reduced to $6 \%$ allowing timing measurements to be used to separate background from the recoiling protons. The detector operates in counting mode. The A4 experiment at Mainz also operates in counting mode. All other electron parity violation experiments operate in integrating mode.

The results [8] are shown graphically in fig. 4 together with the results from other experiments. The results indicate a non-zero, $Q^{2}$-dependent, strange quark contribution. They cover a much broader $Q^{2}$ range than the other experiments. The uncertainties for the higher $Q^{2}$ points are quite large as a result of significant unanticipated background contributions.

G0 is planning to make backward angle measurements in 2006. At backward angles, scattered electrons are detected instead. Each measurement will be at a single $Q^{2}$. They expect to make the first measurements at approximately 0.2 and $0.6(\mathrm{GeV} / c)^{2}$.

\subsection{Neutron densities}

Charge radii of nuclei are well known. For example, the proton radius of the lead nucleus, $R_{p}=5.490 \pm .002 \mathrm{fm}$. This is very precise. A very interesting question is, "What is the neutron radius in $\mathrm{Pb}$ "? Experimentally, $R_{n}$ is rather poorly known. Perhaps it is known to $5 \%$ at best, where the best estimates come from theory.

Models of a neutron star posit a solid crust over a liquid core. The lead nucleus is believed by some to have a neutron skin. Both a possible neutron skin and a neutron star crust would be made of neutron rich matter at similar densities. A measurement of $R_{n}$ to $1 \%$ accuracy would have a major impact on nuclear theory, neutron star structure and atomic parity violation. Currently atomic parity violation experiments are limited in accuracy by our knowledge of the neutron radius in a heavy nucleus.

Donnelly, Dubach and Sick [9] suggested the possibility of using parity violating electron scattering to measure
$R_{n}$. The electromagnetic coupling to the protons is given by $Q_{E M}^{p}=1$ while the neutrons couple by $Q_{E M}^{n}=0$. In contrast the weak coupling of the protons is given by $Q_{W}^{p} \approx 1-4 \sin ^{2} \theta_{W}$ and the neutrons by $Q_{W}^{n} \approx 1$. The neutrons have a very strong weak coupling to the electromagnetic probe. The resulting parity-violating asymmetry is given by

$$
A_{P V}=\frac{-G_{F} Q^{2}}{4 \pi \alpha \sqrt{2}} \frac{F_{W}\left(Q^{2}\right)}{F_{\gamma}\left(Q^{2}\right)},
$$

where

$$
F_{W}\left(Q^{2}\right)=\left(1-4 \sin ^{2} \theta_{W}\right) F_{p}\left(Q^{2}\right)-N F_{n}\left(Q^{2}\right),
$$

and

$$
F_{\gamma}\left(Q^{2}\right)=Z F_{p}\left(Q^{2}\right)
$$

A measurement of $A_{P V}$ to $3 \%$ would provide a measurement of $R_{n}$ to $1 \%$. An experiment has been approved at JLAB to carry out such a measurement. An $850 \mathrm{MeV}$, $50 \mu \mathrm{A}$ polarized beam would be incident on a lead target sandwiched between diamond sheets for cooling. Electrons scattered through $6^{0}$ would be detected in the pair of HallA high-resolution spectrometers. At $Q^{2}=0.01(\mathrm{GeV} / c)^{2}$ the parity-violating asymmetry, $A_{P V}=0.5 \mathrm{ppm}$.

This is a very challenging experiment. It will require control of helicity correlated systematics to much better than $15 \mathrm{ppb}$. It appears to be feasible.

\subsection{Standard Model tests}

Parity violating electron scattering has been used to test the Standard Model (SM) since the pioneering SLAC experiment in the mid-70s. Collider experiments at the Zpole provide our best measure of the weak mixing angle, $\sin ^{2} \theta_{W}$. There remains much interest in exploring the running of $\sin ^{2} \theta_{W}$ from the Z-pole to $Q^{2}=0$.

At $Q^{2}=0$, atomic parity experiments give results consistent with the running of $\sin ^{2} \theta_{W}$. Other experiments testing the $\mathrm{SM}$ include ${ }^{12} \mathrm{C}(\boldsymbol{e}, e),{ }^{9} \mathrm{Be}(\boldsymbol{e}, e), \mathrm{NuTeV}$, Moeller scattering and $Q_{\text {weak }}$. All parity violating experiments can be described in terms four fundamental quark coupling constants,

$$
\begin{aligned}
L_{P V}=-\frac{G_{F}}{\sqrt{2}} & {\left[\bar{e} \gamma_{\mu} \gamma_{5} e\left\{C_{1 u} \bar{u} \gamma^{\mu} u+C_{1 d} \bar{d} \gamma^{\mu} d\right\}+\right.} \\
& \left.+\bar{e} \gamma_{\mu} e\left\{C_{2 u} \bar{u} \gamma^{\mu} \gamma^{5} u+C_{2 d} \bar{d} \gamma^{\mu} \gamma^{5} d\right\}\right]
\end{aligned}
$$

The SM makes predictions for the constants. These constants in turn can be written in terms of isoscalar and isovector combinations at the hadronic vertex:

$$
\begin{gathered}
\tilde{\alpha}=-C_{1 u}+C_{1 d}=-\left(1-2 \sin ^{2} \theta_{W}\right), \\
\tilde{\beta}=-C_{2 u}+C_{2 d}=-\left(1-4 \sin ^{2} \theta_{W}\right), \\
\tilde{\gamma}=-C_{1 u}-C_{1 d}=2 / 3 \sin ^{2} \theta_{W}, \\
\tilde{\delta}=-C_{2 u}-C_{2 d}=0, \\
\sin ^{2} \theta_{W}=0.23120 \pm 0.00015 .
\end{gathered}
$$




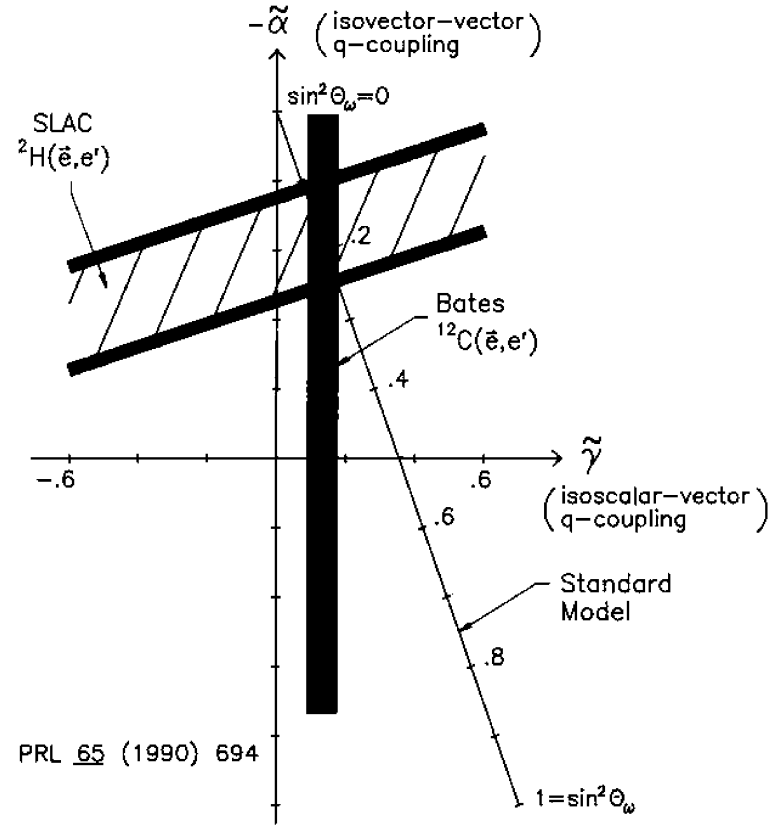

Fig. 6. Results for the SLAC and Bates parity violation experiments in the space of the coupling constants $\tilde{\alpha}$ and $\tilde{\gamma}$.

1. ${ }^{12} \mathrm{C} /{ }^{9} \mathrm{Be}$

${ }^{12} \mathrm{C}$ is a spinless and isoscalar nucleus and elastic electron scattering is described by a single form factor. The parityviolating asymmetry may be written at the tree level $[10$, 11] as

$$
A_{P V}=\tilde{\gamma} \frac{3}{2} G_{F} Q^{2}(\sqrt{2} \pi \alpha)^{-1} .
$$

A parity violation experiment was carried out at MITBates $[12]$ on ${ }^{12} \mathrm{C}$. The results are shown in fig. 6 together with the results of the SLAC experiment [13] on deuterium. The results of both experiments are consistent with the predictions of the SM.

\section{2. $\mathrm{E}-158$}

A recent experiment testing the SM was E-158 at SLAC. It is a purely leptonic process involving Moeller scattering. The goal of the experiment was to measure the weak charge of the electron, $e=g \sin \theta_{W}$, using parity violation.

In this experiment $50 \mathrm{GeV}$ electrons were incident on a liquid hydrogen target. At $Q^{2}=0.027(\mathrm{GeV} / c)^{2}$ the parity violating asymmetry is expected to be $150 \mathrm{ppb}$. A quadrupole spectrometer was used to focus the scattered Moeller electrons while at the same time defocusing e-p scattering events. The flux of scattered electrons was integrated for each beam burst. The experiment yielded an asymmetry,

$$
A_{P V}=-175 \pm 30 \pm 20 \mathrm{ppb} .
$$

The extracted weak mixing angle is totally consistent with predictions. The result [14] is shown plotted in fig. 8 . 3. $\mathrm{Q}_{\text {weak }}$

A major new initiative is under development at JLAB. The goal of the Qweak experiment is to measure the pro-

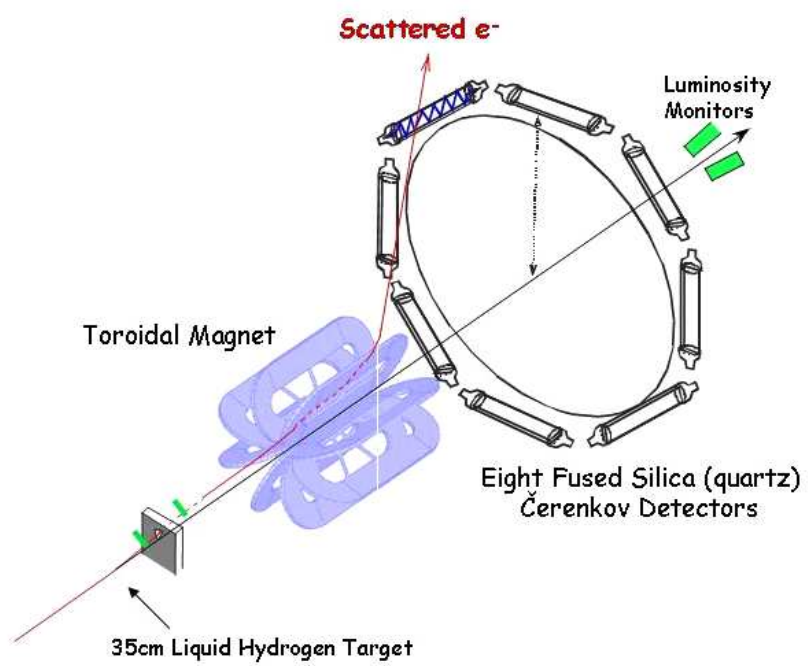

Fig. 7. Schematic layout of the $\mathrm{Q}_{\text {weak }}$ experiment showing the target, collimators, shielding, toroidal magnet and detector system.

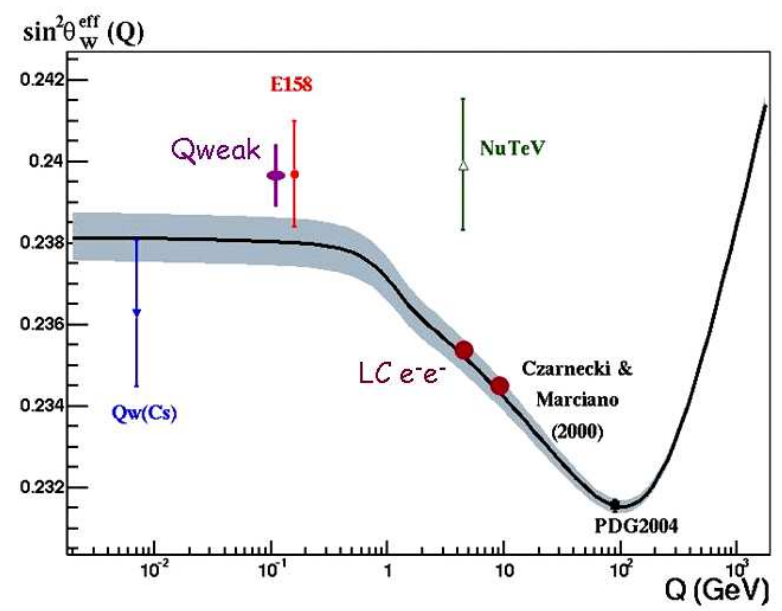

Fig. 8. Calculated running of the weak mixing angle in the Standard Model. Data points are from the atomic parity violation experiment on $\mathrm{Cs}$, the $\mathrm{NuTeV}$ experiment, the Moeller experiment (E-158) at SLAC and from experiments at the $\mathrm{Z}^{0}$ pole. Also shown are the anticipated error bars for $Q_{\text {weak }}$.

ton's weak charge, $Q_{W}^{p}=1-4 \sin ^{2} \theta_{W}$, to the highest precision possible.

The SM makes a firm prediction of $Q_{W}^{p}$ based on the running of the weak mixing angle $\sin ^{2} \theta_{W}$ from the $\mathrm{Z}^{0}$ pole to low energies, corresponding to a $10 \sigma$ effect in our experiment. Fig. 8 shows the SM prediction for $\sin ^{2} \theta_{W}$ together with existing data and the expected precision for this experiment. This parity violating experiment is in the semi-leptonic sector. This is in contrast to E-158 which is in the purely leptonic sector.

The measurement will be carried out using a $1.2 \mathrm{GeV}$ electron beam at a scattering angle of $9^{\circ}$ and a momentum 
transfer $Q^{2}=0.03(\mathrm{GeV} / c)^{2}$. The $180 \mu \mathrm{A}$ polarized beam will be incident on a $35 \mathrm{~cm}$ liquid hydrogen target. An eight sector toroidal spectrometer is being constructed for this measurement. The scattered electrons will be detected by quartz Cherenkov detectors operating in integrating mode. A schematic layout of the spectrometer is shown in fig. 7 .

The measurement will take 2200 hours and will determine the proton's weak charge with $4 \%$ statistical accuracy. Figure 8 shows the projected quality of the $Q_{\text {weak }}$ results in the context of other existing data.

\section{Summary}

Parity-violating high-energy electron scattering is an important probe of nucleon and nuclear structure. Several physics areas are currently under investigation. There have been many experiments investigating the importance of strange quarks to the structure of the proton. Results to date indicate that the contribution of strange quarks to nucleon structure are relatively small or vanishing. A proposed experiment on $\mathrm{Pb}$ would measure the neutron radius to $1 \% . Q_{\text {weak }}$ is a challenging Standard Model test which probes $5 \mathrm{TeV}$ energy scales.
Such demanding measurements have been made possible by important advances in accelerator technology. We now have high intensity $\mathrm{CW}$ beams with beam polarization of $85 \%$. Control of helicity correlated beam properties allows measurement of asymmetries to an accuracy approaching 10's ppb.

\section{References}

1. R.D. McKeown, Phys. Lett. B 219, 140 (1989).

2. D. Keyslan, A. Manohar, Nucl. Phys. B 310, 527 (1988).

3. M.J. Musolf et al., Phys. Rep. 239, 1 (1994).

4. D.T. Spangle et al., Phys. Lett. B 583, 79 (2004).

5. K.A. Aniol et al., Phys. Rev. C 69, 065501 (2004).

6. The HAPPEX Collaboration (K.A. Aniol et al.), Phys. Lett. B 635, 275 (2006), nucl-ex/0506011.

7. The HAPPEX Collaboration (K.A. Aniol et al.), Phys. Rev. Lett. 96, 022003 (2006), nucl-ex/0506010.

8. D.S. Armstrong et al., Phys. Rev. Lett. 95, 092001 (2005).

9. T.W. Donnelly et al., Nucl. Phys. A 503, 589 (1989).

10. G. Feinberg, Phys. Rev. D 12, 3575 (1975).

11. J.D. Walker, Nucl. Phys. A 285, 345 (1977).

12. P.A. Souder et al., Phys. Rev. Lett. 65, 694 (1990).

13. C.Y. Prescott et al., Phys. Lett. B 84, 524 (1979).

14. P.L. Anthony et al., Phys. Rev. Lett. 95, 081601 (2005). 